\title{
DNER modulates the length, polarity and synaptogenesis of spiral ganglion neurons via the Notch signaling pathway
}

\author{
JINTAO DU ${ }^{1 *}$, XIANREN WANG ${ }^{2 *}$, XIAOBO ZHANG ${ }^{2}$, XUEMEI ZHANG ${ }^{2}$ and HONGYAN JIANG ${ }^{2}$ \\ ${ }^{1}$ Department of Otorhinolaryngology Head \& Neck Surgery, West China Hospital, Sichuan University, \\ Chengdu, Sichuan $610041{ }^{2}$ Department of Otorhinolaryngology, The First Affiliated Hospital, \\ Sun Yat-sen University, Guangzhou, Guangdong 510080, P.R. China
}

Received February 24, 2016; Accepted February 16, 2017

DOI: $10.3892 / \mathrm{mmr} .2017 .8115$

\begin{abstract}
The Delta/Notch-like epidermal growth factor-related receptor (DNER) serves an important role in the developing central nervous system. However, the actions of DNER in the development of the spiral ganglion in the inner ear have yet to be elucidated. Wild-type C57BL/6 mice were housed and time-mated for use in the present study. Primary neuronal cultures were prepared using spiral ganglion progenitors isolated from the modiolus of postnatal day 1 (P1) mice. DNER recombinant lentiviral vectors were constructed and transfected into the cultured primary neurons. The relative proportion of differentiated neurons and the length of their neurites were evaluated using microscopy. The results of the present study demonstrated that DNER was expressed in spiral ganglion neurons (SGNs) that exhibited significant polarity in the early differentiation stages; DNER expression gradually decreased until the polarity was lost on week 35 . The in vitro expression of DNER was revealed to be similar to that in vivo. When DNER expression was silenced using RNA interference, the polarity of the differentiated neurons was altered and they exhibited significantly reduced dendritic length. In addition, the proportion of bipolar neurons was decreased compared with the control group. Furthermore, the expression of $\alpha$-synuclein and the GluR2/3 subunits of the $\alpha$-amino-3-hydroxy-5-methyl-4-isoxazolepropionic acid glutamate receptor were also reduced in cultured neurons in which DNER was silenced. Notch1 was co-expressed with DNER in SGNs isolated from P1 mice. The indirect Notch inhibitor N-[N-(3,5-Difluorophenacetyl)-L-alanyl]-S-phenylglycine
\end{abstract}

Correspondence to: Professor Hongyan Jiang, Department of Otorhinolaryngology, The First Affiliated Hospital, Sun Yat-sen University, 58 Zhongshan Road, Guangzhou, Guangdong 510080, P.R. China

E-mail: jhongy@mail.sysu.edu.cn

*Contributed equally

Key words: DNER, neuronal polarity, neuritogenesis, spiral ganglion neurons, Notch t-butyl ester also affected the polarity and the formation of protrusions, and reduced the expression of DNER and glial fibrillary acidic protein in SGNs. In conclusion, the present study demonstrated that DNER was expressed in SGNs and appeared to be involved in the mechanisms underlying neuronal polarity and neuritogenesis, via a Notch-dependent signaling pathway.

\section{Introduction}

A decrease in the number of spiral ganglion neurons (SGNs), abnormal dendritic growth and impairments in polarity have been associated with sensorineural hearing loss, neural tinnitus and unsatisfying electronic cochlear implant (CI) results (1). Strategies to improve the effects of CI include promoting the survival of SGNs, as well as potentiating the growth of functional fibers from residual SGNs $(1,2)$. Nerve fiber growth is regulated by neuritogenesis, polarity development and synapse formation. Therefore, exploring the mechanisms underlying these events in SGNs may yield potential therapeutic strategies to improve the outcome for patients undergoing CI treatment.

Notch signaling is implicated in the mechanisms underlying the development of several tissues, including the inner ear. Previous studies have reported roles for Notch signaling within multiple steps of the generation and differentiation of inner ear structures through various pathways (3-6). The Delta/Notch-like epidermal growth factor (EGF)-related receptor (DNER) is a single-pass transmembrane protein with 10 EGF-like repeats in its extracellular domain. The EGF-like repeats in the DNER structure are similar to those found in the structures of Notch and its ligands Delta and Jagged $(7,8)$. DNER expression has been reported in the olfactory bulb (8), the developing and mature central nervous system (CNS) (7-12), and the inner ear (13-15). DNER has been implicated in neural progenitor development, neural proliferation, and neuronal and glial differentiation via Notch-dependent and Notch-independent pathways $(9,10,16)$. Notably, Hartman et al (13) reported a robust expression pattern for DNER in developing and mature neurons and hair cells in the inner ear, and also demonstrated that supporting cells and glia appeared normal in the inner ear of adult $\mathrm{DNER}^{-/-}$mice. However, a study by Kowalik and Hudspeth (14) suggested that DNER and protein-tyrosine-phosphatase (PTP) $\zeta$ may control 
hair-bundle morphology in order to establish the tonotropic gradient between the high- and low-frequency regions of the chick cochlea. Further physiological and comprehensive morphological studies of auditory and vestibular function are required to elucidate the putative roles of DNER in neurons and hair cells of the inner ear.

Pleiotrophin-PTP $\zeta$ signaling has been implicated in the control of the subcellular localization of DNER in cerebellar Purkinje cells and in the Neuro-2a cell line, and has therefore been suggested to regulate neuritogenesis (16). Furthermore, DNER has been reported to induce Bergmann glial differentiation during astrocytogenesis in the CNS, and to inhibit myotube differentiation in $\mathrm{C} 2 \mathrm{C} 12$ myoblasts in vitro (10). Therefore, the roles of DNER in the development and maturation of the spiral ganglion may be complex and require further investigation. Previous studies $(13,14)$ have revealed that DNER was robustly expressed in the inner ear, including inSGNs. The results of the present study suggested that DNER may participate in polarization and neurite extension processes in SGNs and may exert its actions via the Notch signaling pathway.

\section{Materials and methods}

Mouse husbandry. A total of 20 female and 10 male wild-type C57BL/6J mice were propagated and housed in the Experimental Animal Center of Sun Yat-sen University (Guangzhou, China) under specific pathogen-free condition and allowed free access to sterile water and food with a 12:12-h light/dark cycle (lights on at 6:00 a.m. and off at 6:00 p.m.) at $22^{\circ} \mathrm{C}$. The mice were time-mated, and embryonic day 0.5 (E0.5) was defined as noon on the day of the observation of the vaginal plug. The embryos were staged according to the EMAP eMouse Atlas Project (http://www.emouseatlas.org) (17). The Institutional Animal Care and Use Committee of the Sun Yat-sen University approved the experimental methods and animal care procedures.

Primary SGN culture. Primary cells from the cochlear modiolus were cultured as previously reported (18). Briefly, on postnatal day 1 (P1) C57BL/6 mice were sacrificed and cochlear modioli were collected and dissected in Hank's balanced salt solution (pH 7.4; HBSS; Gibco; Thermo Fisher Scientific Inc., Waltham, MA, USA). The cochlear sensory epithelium was removed from the modiolus and the entire modiolus was placed in $0.05 \%$ trypsin/EDTA in HBSS that had been prewarmed to $37^{\circ} \mathrm{C}$ for $5 \mathrm{~min}$. Subsequently, $20 \%$ fetal bovine serum (Gibco; Thermo Fisher Scientific Inc.) was added to terminate the trypsin digestion and a homogeneous single cell suspension was obtained via gentle pipetting. The suspension was centrifuged for $6 \mathrm{~min}$ at $200 \mathrm{x} \mathrm{g}$ at $22^{\circ} \mathrm{C}$ and resuspended in differentiation medium at a concentration of $10^{6}$ cells $/ \mathrm{ml}$. The differentiation medium contained serum-free Dulbecco's modified Eagle's medium-F12 (1:1) (Gibco; Thermo Fisher Scientific Inc.), supplemented with 20 ng/ml neurotrophin-3 (PeproTech China, Suzhou, China), $20 \mathrm{ng} / \mathrm{ml}$ brain-derived neurotrophic factor (PeproTech, Suzhou, China), 2 mM L-glutamine (Gibco; Thermo Fisher Scientific, Inc., Waltham, MA, USA), $10 \mathrm{ng} / \mathrm{ml}$ leukemia inhibitory factor (R\&D Systems, Inc., Minneapolis, MN, USA) and $3 \mathrm{mM} \mathrm{KCl} \mathrm{(Merck} \mathrm{KGaA,} \mathrm{Darmstadt,} \mathrm{Germany).}$
The suspension was passed through a $70-\mu \mathrm{m}$ cell filter and the cells were plated on collagen-coated coverslips in 6-well plates at the density of $2 \times 10^{5} / \mathrm{ml}$ for use in subsequent experiments.

$N$-[N-(3,5-Difluorophenacetyl)-L-alanyl]-S-phenylglycine $t$ butyl ester $(D A P T)$ treatment. Primary cells from the cochlear modiolus prepared as above, were divided into two groups, one as a control group, and the other as the DAPT treated group. DAPT (D5942; Sigma-Aldrich; Merck KGaA, Darmstadt, Germany) at a final concentration of $5 \mu \mathrm{M}$ as in a previous study (19) was added to the culture medium and the cells treated for $24 \mathrm{~h}$ to $48 \mathrm{~h}$ to the time of sample preparation.

Immunofluorescence. Immunostaining was performed as previously described (20) using the following primary antibodies: Goat polyclonal anti-DNER (AF2254; 1:300; R\&D Systems, Inc., Minneapolis, MN, USA), mouse monoclonal anti-neuron-specific class III $\beta$-tubulin (Tuj1; 1:200; MO15013; Neuromics, Inc., Edina, MN, USA), mouse monoclonal anti-activated Notch1 (1:200; ab8925; Abcam, Cambridge, MA, USA), rabbit polyclonal anti- $\alpha$-synuclein (1:200; 2628; Cell Signaling Technology, Inc., Danvers, MA, USA) and rabbit polyclonal anti-GluR2/3 (1:300; AB1506; Chemicon $^{\circledR}$; EMD Millipore, Billerica, MA, USA). Hoechst 33342 (1:1,000; H1399; Invitrogen; Thermo Fisher Scientific, Inc.) was used to stain the nucleus. The following secondary antibodies were used: Alexa Fluor ${ }^{\circledR} 488$-conjugated and Alexa Fluor ${ }^{\circledR}$ 594-conjugated donkey anti-mouse, Alexa Fluor ${ }^{\circledR} 488$-conjugated and Alexa Fluor ${ }^{\circledR}$ 594-conjugated donkey anti-rabbit, and Alexa Fluor ${ }^{\circledR}$ 594-conjugated donkey anti-goat antibodies (1:400; R37114, R37115, R37118, R37119, A-11055 and A-11058 respctively; Invitrogen; Thermo Fisher Scientific, Inc.). Time-mated pregnant mice were sacrificed under isoflurane (4\%) anesthesia by cervical dislocation at E10.5-18.5 and embryonic otocysts were carefully dissected under a stereomicroscope. Embryonic otocysts (E12.5, E14.5, E16.5 and E18.5) and postnatal day 1 and 28, and weeks 6 and 35 inner ear samples were collected and immediately fixed in $4 \%$ paraformaldehyde (PFA)/PBS at $4^{\circ} \mathrm{C}$ overnight. Subsequently, samples were washed three times with PBS and dehydration was performed in graded sucrose. Tissue samples were sliced into $7 \mu \mathrm{m}$ sections using a cryostat and air-dried for $30 \mathrm{~min}$ at room temperature, followed by long-term storage at $-20^{\circ} \mathrm{C}$. Following rehydration in PBS, the sections were permeabilized in $0.1 \%$ Triton $\mathrm{X}-100$ in PBS for $15 \mathrm{~min}$ and washed three times for $5 \mathrm{~min}$ in PBS. Sections were blocked in $5 \%$ bovine serum albumin (AR1006; Boster Biological Technology, Ltd., Wuhan, China) $/ 0.05 \%$ Triton X-100 in PBS for $1 \mathrm{~h}$ prior to incubation with the primary antibodies overnight at $4^{\circ} \mathrm{C}$. Incubation with the secondary antibodies (1:400) was performed at $4^{\circ} \mathrm{C}$ overnight or at room temperature for $2 \mathrm{~h}$. Nuclei were stained with Hoechst $33342(1: 1,000)$ at room temperature for 15 min. Samples were mounted and observed under a fluorescence microscope (Olympus IX71; Olympus Corporation, Tokyo, Japan; or Leica Microsystems, Inc., Buffalo Grove, IL, USA) and a FluoView FV1000 confocal microscope (Olympus Corporation). Cultured SGNs were prepared for staining and fixed on coverslips in 4\% PFA/PBS for $20 \mathrm{~min}$ 
at room temperature and then washed in PBS three times. Subsequently, permeabilization, blocking and antibody incubation were performed as described for tissue samples.

Reverse transcription semi-quantitative polymerase chain reaction ( $R T$-semi-quantitative $P C R$ ). Early-stage otocysts (E14.5, 16.5 and 19) and late-stage cochlear modioli (P1) were carefully dissected and collected in Eppendorf tubes. The cells were collected in Eppendorf tubes at different time points (E14.5, E16.5, E19 and P1). In addition, cultured SGNs treated with DAPT or not at $72 \mathrm{~h}$ were collected to extract total RNA. Total RNA was extracted using the RNeasy purification kit (Qiagen $\mathrm{GmbH}$, Hilden, Germany) according to the manufacturer's protocol. Total RNA was treated with RNase-free DNase (Roche Diagnostics, Indianapolis, IN, USA) and reverse transcribed into cDNA using SuperScript III Reverse Transcriptase (Invitrogen; Thermo Fisher Scientific, Inc.) according to the protocol provided by manufacturer. The qPCR process consists of 1 cycle at $94^{\circ} \mathrm{C}$ for $3 \mathrm{~min}$ followed by 35 cycles at $94^{\circ} \mathrm{C}$ for $30 \mathrm{sec}, 35$ cycles at $55^{\circ} \mathrm{C}$ for $30 \mathrm{sec}$ and $72^{\circ} \mathrm{C}$ for $1 \mathrm{~min}$, followed by $72^{\circ} \mathrm{C}$ for $5 \mathrm{~min}$ with PCR Reagent (KT207-01; Tiangen Biotech Co., Ltd., Beijing, China). The following primers were used: DNER, forward 5'-GCCGCC TTTGTGCTTCTGTTC-3', reverse 5'-CCGGTGGTCTGT CTGGTCGTC-3'; glial fibrillary acidic protein (GFAP), forward 5'-GGGGCAAAAGCACCAAAGAAG-3', reverse 5'-GGGACAACTTGTATTGTGAGCC-3'; and GAPDH, forward 5'-GAAGGTGAAGGTCGGAGTC-3', reverse 5'-GAAGATGGTGATGGGATTTC-3'. The PCR products were resolved by $1 \%$ agarose gel electrophoresis containing $0.4 \mu \mathrm{g} / \mathrm{ml}$ of ethidium bromide. The level of gene expression was semi-quantified by grey scale ratio of DNER vs. GAPDH by Quantity One (version 462; Bio-Rad Laboratories, Inc. Hercules, CA, USA). The experiment was independently performed at least three times with at least four otocysts or cochleae from each group.

Western blot analysis. Dissected cochlear modioli from E14.5, E18.5 and P1 mice and the left hemisphere of the brains from P1 neonatal mice were washed twice in ice-cold PBS, resuspended and homogenized in lysis buffer containing $50 \mathrm{mM}$ Tris- $\mathrm{HCl}$, $\mathrm{pH} 7.4,1 \% \mathrm{NP}-40,10 \%$ glycerol, $150 \mathrm{mM} \mathrm{NaCl}, 11 \mathrm{mM}$ sodium orthovanadate, $1 \mathrm{mM}$ phenylmethylsulfonyl fluoride and a protease inhibitor cocktail (Roche Diagnostics). Homogenates were centrifuged at $1,000 \mathrm{x}$ g for $5 \mathrm{~min}$ at $4^{\circ} \mathrm{C}$, and supernatants were collected. Protein concentration was determined by Eppendorf BioSpectrometer ${ }^{\circledR}$ basic at UV $280 \mathrm{~nm}$ (Eppendorf, Hamburg, Germany). Equal amounts of extracted protein $(20 \mu \mathrm{g})$ were separated by $8-15 \%$ SDS-PAGE, transferred onto polyvinylidene fluoride membranes which were immersed into methanol for $10 \mathrm{sec}$ at room temperature and incubated with the following antibodies: Anti-DNER (1:1,000; AF2254, R\&D Systems, Inc., Minneapolis, MN, USA), anti-Notch1 (ab8925; 1:2,000; Abcam, Cambridge, MA, USA) and anti-GAPDH (1:3,000; sc-47724, Santa Cruz Biotechnology, Inc., Dallas, TX, USA) at $4^{\circ} \mathrm{C}$ overnight, according to the manufacturer's protocol. After washing 3 times in TBST each for $5 \mathrm{~min}$, the membranes were incubated for $2 \mathrm{~h}$ at room temperature with secondary antibodies goat anti-rabbit and mouse immunoglobulin G/horseradish peroxidase (bs-0295G-HRP and
bs-0296G-HRP; Beijing Biosynthesis Biotechnology Co., Ltd., Beijing, China). After extensive washes, chemiluminescence was used to visualize the expression of protein with a Novex ${ }^{\circledR}$ ECL Chemiluminescent Substrate Reagent kit (WP20005; Invitrogen, Thermo Fisher Scientific, Inc.) as according to the manufacturer's protocols.

Construction of DNER recombinant lentivirus and cell transfection. According to the mRNA sequence of DNER available on GenBank (NM_139072; https://www.ncbi.nlm. nih.gov/genbank/), the following pair of target sequences of short hairpin (sh)RNA met the requirements of the BLOCK-iT Lentiviral RNA interference (RNAi) expression system (Invitrogen; Thermo Fisher Scientific, Inc.): shRNA1, 745'-CGACGATTGTCCAGGAAACAA-3' 794; shRNA2, 2241 5'-GACCAACTGTGACATCAACAA-3' 2261. The following two pairs of complementary stem-loop structures and one pair of negative control structures encoding the shRNA target sequences were designed and synthesized: DNER shRNA1, top strand 5'-caccGTTGTTTCCTGGACAAT CGTCGcgaaCGACGATTGTCCAGGAAACAA-3', bottom strand 5'-aaaaTTGTTTCCTGGACAATCGTCGttcgCGAC GAT TGTCCAGGAAACAAC-3'; DNER shRNA2, top strand 5'-caccGTTGTTGATGTCACAGTTGGTCcgaaGAC CAACTGTGACATCAACAA-3', bottom strand 5'-aaaaTT GTTGATGTCACAGTTGGTCttcgGACCAACTGTGACAT CAACAAC-3'; negative control shRNA, top strand 5'-caccGT TCTCCGAACGTGTCACGTcgaaACGTGACACGTTCG

GAGAA-3', bottom strand 5'-aaaaTTCTCCGAACGTGT CACGTttcgACGTGACACGTTCGGAGAAC-3'. (The lower case letters in the top strand oligo, 'cacct', at the 5' end of the oligo is complementary to the overhang sequence, GTGG, in the $\mathrm{pENTR}^{\mathrm{mi}} / \mathrm{U} 6$ vector and constitutes the last 4 bases of the U6 promoter. The lower case letters in bottom strand oligo 'aaaa' at the 5' end of the oligo is complementary to the overhang sequence, TTTT, in the $\mathrm{pENTR}^{\mathrm{TM}} / \mathrm{U} 6$ vector and constitutes the first 4 bases of the Pol III terminator. While the lower-case letters in the middle of oligos indicated the loop structure.) The single-stranded oligonucleotides were annealed to create double-stranded oligonucleotides, which were cloned into the $\mathrm{pENTR}^{\mathrm{TM}} / \mathrm{U} 6$ vector $\left(\mathrm{BLOCK}^{\mathrm{iT}}{ }^{\mathrm{TM}} \mathrm{U} 6\right.$ RNAi Entry Vector Kit; Invitrogen; Thermo Fisher Scientific, Inc.) using an optimized 5-min ligation procedure. Competent E. coli cells (kept and propagated in the Laboratory of Otorhinolaryngology, the First Affiliated Hospital of Sun Yat-sen University, Guangzhou, China) were transformed and selected for entry clones. A pLenti6/BLOCK-iT ${ }^{\mathrm{TM}}$-DEST vector (Thermo Fisher Scientific, Inc.) and a $\mathrm{pENTR}^{\mathrm{TM}} / \mathrm{U} 6$ entry clone with the ds oligo encoding the shRNA of DNER were used in a Gateway LR recombination reaction according to the manufacturer's protocols to generate an expression clone containing the U6 RNAi cassette of interest. The pLenti6/BLOCK- iT ${ }^{\mathrm{TM}}$-DEST expression construct and the ViraPower $^{\mathrm{TM}}$ Lentiviral Packaging Mix (Thermo Fisher Scientific, Inc.) were co-transfected into the 293FT cell line (Thermo Fisher Scientific, Inc.) to produce a lentiviral stock. Viruses were collected from these cells and stored at $-80^{\circ} \mathrm{C}$. Viral titers were averaged and typically ranged between $1-5 \times 10^{8} \mathrm{IU} / \mathrm{ml}$. Briefly, 293T cells were cultured in 10 wells of 96 -well plate at the density of $1 \times 10^{5} / \mathrm{ml}$ for 

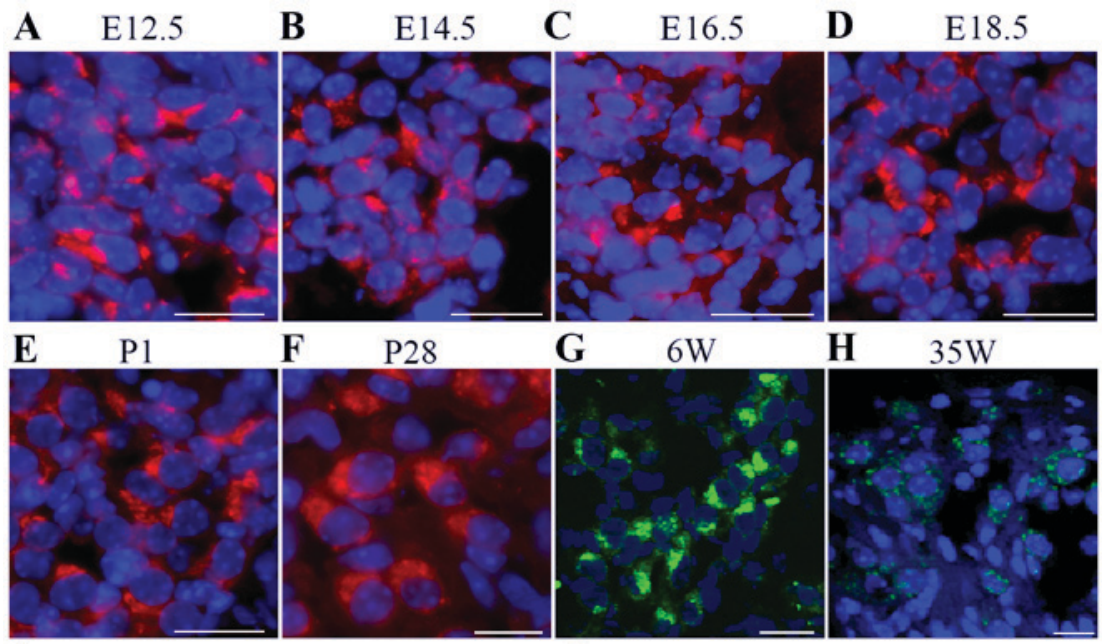

F $\quad$ P28

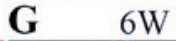

H

$35 \mathrm{~W}$
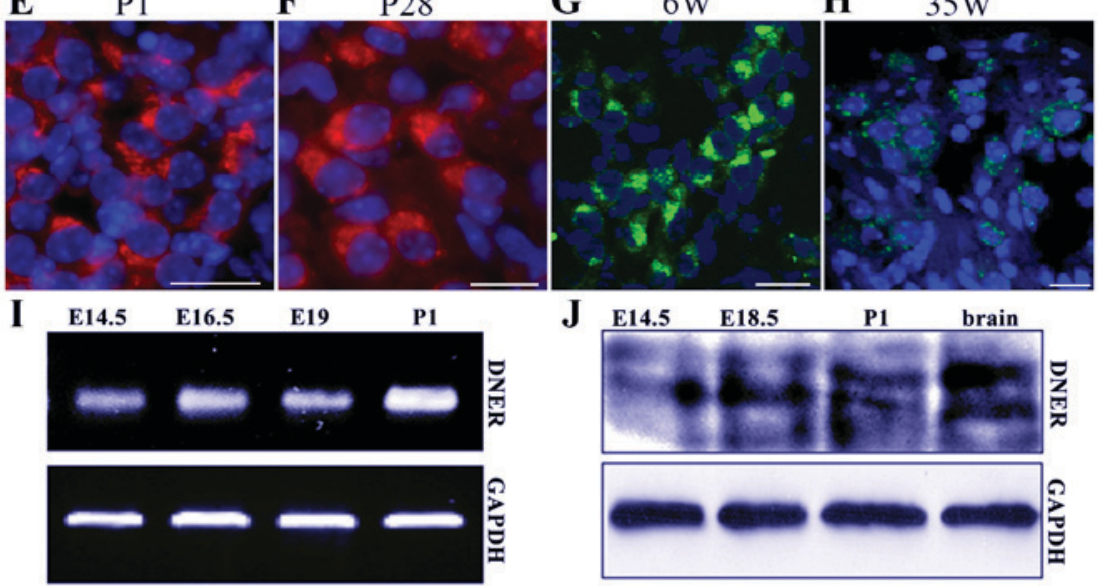

Figure 1. DNER was expressed in spiral ganglion neurons with temporospatial specificity. (A-H) DNER expression in neurons of the developing (E12.5 to P1), adult (P28) and aged spiral ganglion (W35). DNER expression indicated by red in (A-F) and green in (G-H). Nuclei indicated by blue. (I) DNER mRNA expression levels in the inner ear increased during development, as assessed using polymerase chain reaction. (J) DNER protein expression levels in the inner ear increased during development and DNER was robustly expressed in brains of P1 mice. Scale bars, $20 \mu \mathrm{m}$. DNER, Delta/Notch-like epidermal growth factor-related receptor; E, embryonic day; P, postnatal day; W, week.

$24 \mathrm{~h}$. Then 10-fold serial dilution virus solution were added to the 10 wells respectively, and incubated until day 5. The number of cells with green-fluorescent protein (GFP) expression in the last two wells with observable GFP expression were counted as $\mathrm{X}$ and $\mathrm{Y}$ under a fluorescence microscope. The primary virus titer (IU/ml) was determined by $(\mathrm{X}+\mathrm{Yx} 10)$ $\mathrm{x} 1000 / 2$ /volume (original virus solution in the well with the $\mathrm{X}$ value). Lentiviral stocks were used to transduce the cultured SGNs and perform transient RNAi analysis or generate a stably transduced cell line. Viral transduction was performed twice with multiplicity of infection $\sim 50$.

Neuronal populations and neurite length. Transduced SGNs were fixed and immunostained at various post-differentiation time points (48 and $72 \mathrm{~h}$ ), and observed under an Olympus IX71 fluorescent microscope (Olympus Corporation). Experiments were independently repeated three times under identical conditions. To assess neurite growth, the entire length of the longest neurite extending from each differentiated neuron (as identified by Tuj1) was measured within 30 high-power randomly selected visual fields (under a 40x objective). Neurites that were not entirely in the frame were excluded. Neuronal processes that could not be distinguished from others were eliminated. Neurite length was measured using Image-Pro Plus software version 6.0 (Media Cybernetics Inc., Rockville, MD, USA). The relative proportions of the various neuronal populations were determined in 10 randomly selected low-power fields (under a 10x objective). All neurons within the image frame were counted by Image-Pro Plus software version 6.0 (Media Cybernetics
Inc.). Cells that were located in clumps and could not be clearly observed were excluded.

Statistical analysis. The statistical significance of the differences between the control and experimental groups was assessed by Student's t-test. $\mathrm{P}<0.05$ was considered to indicate a statistically significant difference. The analysis was performed using SPSS software version 13.0 (SPSS, Inc., Chicago, IL, USA).

\section{Results}

DNER expression in the developing and mature mouse inner ear. DNER expression has previously been detected in hair cells and SGNs of the inner ear $(13,14)$. The results of the present study demonstrated that DNER was first expressed in developing SGNs from E12.5 to P1, and maintained in P28 and 35-week-old adult mice (Fig. 1A-H). During the early developmental stages, between E12.5 and E18.5, neuronal DNER expression was not characterized by significant polarity. However, in later developmental stages and in adult mice, between P1 and P28, DNER expression was localized in one neuronal pole, and significant polarity was apparent in 6-week-old mice. SGN loss and degeneration in the inner ear appeared on week 35, with a decreasing number of neurons appearing in the in spiral ganglion, at which time DNER expression in SGNs also appeared to decrease and lose its polarity (Fig. 1H). Furthermore, RT-semi-quantitative PCR and western blot analysis revealed that DNER expression increased with inner ear development and peaked on P1 


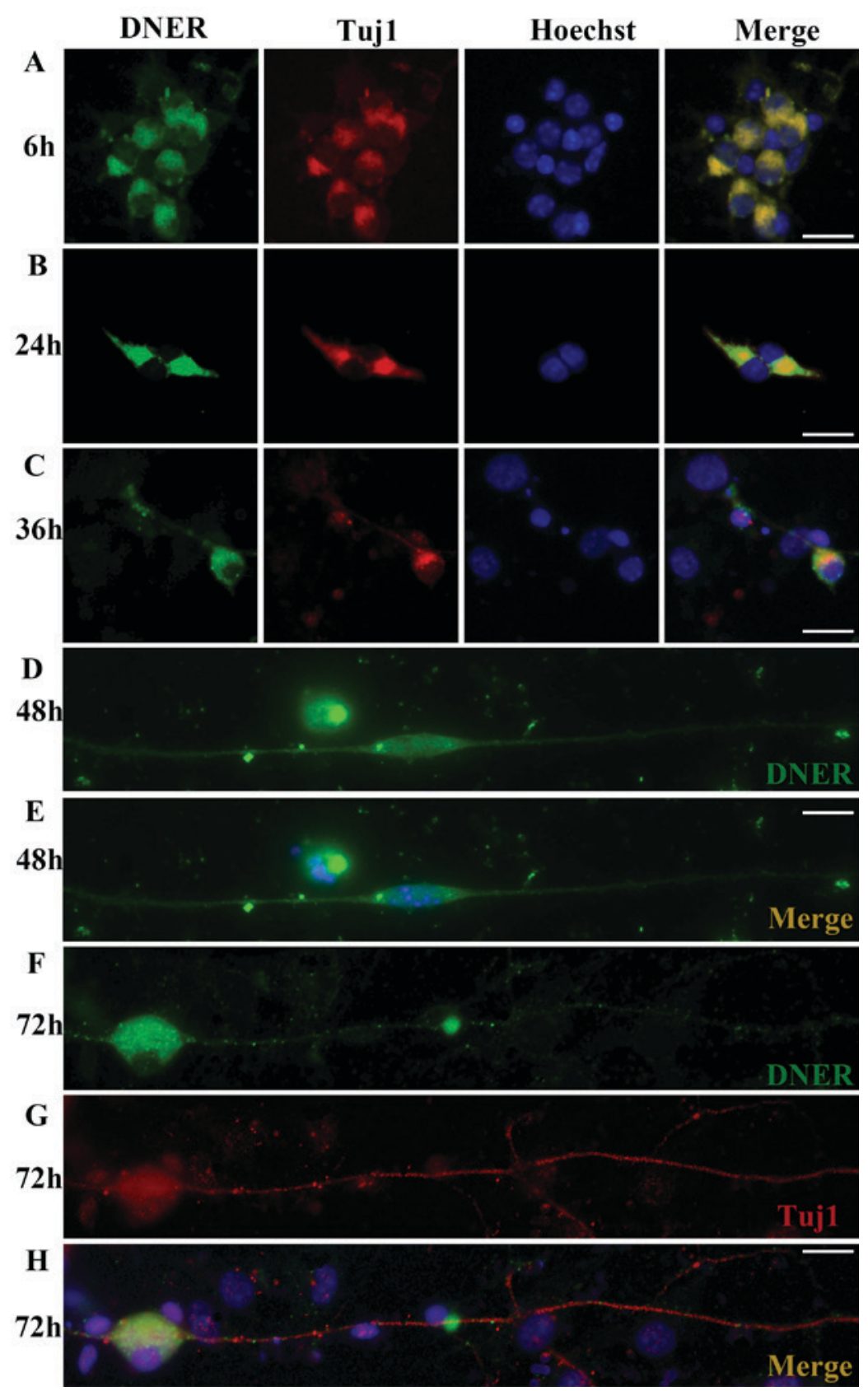

Figure 2. DNER expression in cultured SGNs. (A) DNER was robustly expressed in the somatoplasm and small processes. (B and C) DNER distribution in cultured SGNs exhibited polarity between 24 and $36 \mathrm{~h}$ in culture. (D-H) DNER expression in SGN dendrites was dispersed and punctated following $48 \mathrm{~h}$ of culture and ultimately lost its polarity. DNER, Delta/Notch-like epidermal growth factor-related receptor; SGN, spiral ganglion neuron; Tuj1, neuron-specific class III $\beta$-tubulin. Scale bar, $20 \mu \mathrm{m}$.

(Fig. 1I and J). In accordance with previous studies (7-10), DNER was also observed to be markedly expressed in the brain (Fig. 1J).

DNER expression in cultured SGNs. Inner ear modioli isolated from P1 mice were used to generate primary neural progenitor cultures. DNER expression was observed in undifferentiated neurons and was characterized by an apparently polarized distribution. Following in vitro culture for $6 \mathrm{~h}$, protrusions formed on one pole of the SGNs, and DNER appeared robustly expressed in the somatoplasm and in small processes (Fig. 2A). As culture duration increased (24, 36 and 48 h; Fig. 2B-D), SGN protrusions developed into dendrites or axons, and
DNER expression was observed in these processes. However, at longer culture times (72 h; Fig. 2F-H), dendritic DNER expression appeared punctate and dispersed, and ultimately lacked polarity.

DNER silencing affects polarity and protrusion formation in $S G N s$. DNER expression was silenced in spiral neuron precursor cells in vitro. The expression of DNER was almost completely inhibited following $48 \mathrm{~h}$ of SGN culture and the number of differentiated neurons was significantly decreased. Neurons in the DNER knockdown group were primarily monopolar or multipolar (Fig. 3), whereas the control group was dominated by bipolar neurons (Fig. 2). Furthermore, 

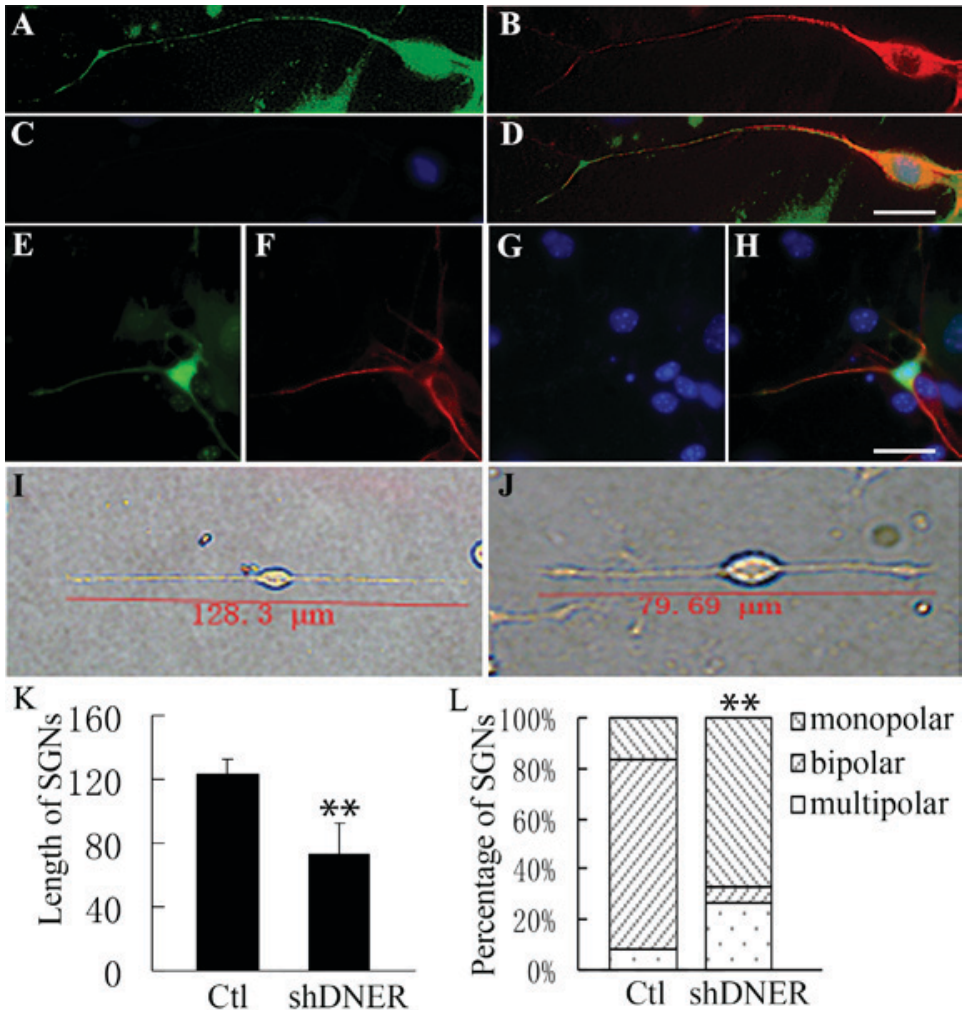

Figure 3. Neuronal polarity was affected during neural progenitor development following DNER knockdown in vitro. Neural progenitors developed into (A-D) unipolar or (E-H) multipolar neurons following DNER knockdown. (A and E) Green indicates GFP expression accompanied by the DNER RNAi via the Block iT ${ }^{\text {Th }}$ Lentiviral RNAi expression system. GFP indicates the knockdown of DNER. (B and F) Neuron-specific class III $\beta$-tubulin is presented in red. (C and G) Nuclei stained with Hoechst 33342 are presented in blue. Merged images (D) from A, B and C or (H) from E, F and G. (I-J) Compared with (I) the control group, neurons (J) not expressing DNER exhibited markedly shorter dendrites. (K) SGN length following DNER knockdown was significantly shorter compared with the control group. ${ }^{* *} \mathrm{P}<0.01$. Bars indicate mean \pm standard deviation. (L) Following DNER knockdown, spiral ganglia neural progenitors gave rise to a significantly lower proportion of bipolar neurons compared with the control group. ${ }^{* *} \mathrm{P}<0.01$. Stacked bars indicated the mean proportion of neuron types. Scale bar, $20 \mu \mathrm{m}$. DNER, Delta/Notch-like epidermal growth factor-related receptor; GFP, green fluorescent protein; SGN, spiral ganglion neuron; sh, short hairpin; Ctl, control.

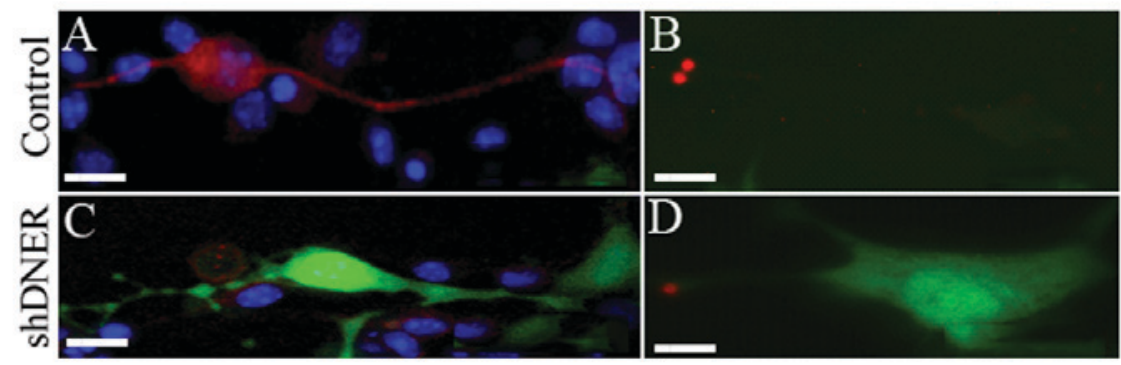

Figure 4. $\alpha$-synuclein and GluR2/3 expression is reduced in neurons lacking DNER compared with the control group. (A and B) Control spiral gangli shDNER on neurons exhibited normal $\alpha$-synuclein and GluR2/3 expression. (C and D) Following DNER knockdown, the expression of $\alpha$-synuclein and GluR2/3 was reduced compared with in the control group. Red is (A and C) $\alpha$-synuclein or (B and D) GluR2/3. Green fluorescent protein is presented in green and indicates the knockdown of DNER. Scale bar, $20 \mu \mathrm{m}$. DNER, Delta/Notch-like epidermal growth factor-related receptor.

neurons not expressing DNER sprouted markedly shorter dendrites $(79.69 \pm 19.6 \mu \mathrm{m})$ compared with control neurons $(123.8 \pm 9.1 \mu \mathrm{m}$; Fig. 3I-K). In addition, DNER knockdown resulted in reduced expression of $\alpha$-synuclein and GluR2/3 in SGNs compared with in the control group (Fig. 4).

$N$-[N-(3,5-Difluorophenacetyl)-L-alanyl]-S-phenylglycine t-butyl ester (DAPT)affects SGN polarity and protrusion formation similar to DNER knockdown. During the maturation of spiral ganglion progenitors, Notch1 is cleaved by $\gamma$-secretase to produce its active form, active-Notch1 (3), which was revealed to be co-expressed with DNER in SGNs from P1 mice (Fig. 5A). Treatment with the $\gamma$-secretase inhibitor DAPT $(5 \mu \mathrm{M})$ suppressed the activation of Notch in cultured neurons. Compared with neurons where DNER was knocked down, spiral ganglion progenitors treated with DAPT exhibited impaired differentiation, and the proportion of monopolar neurons was greater among DAPT-treated cells 


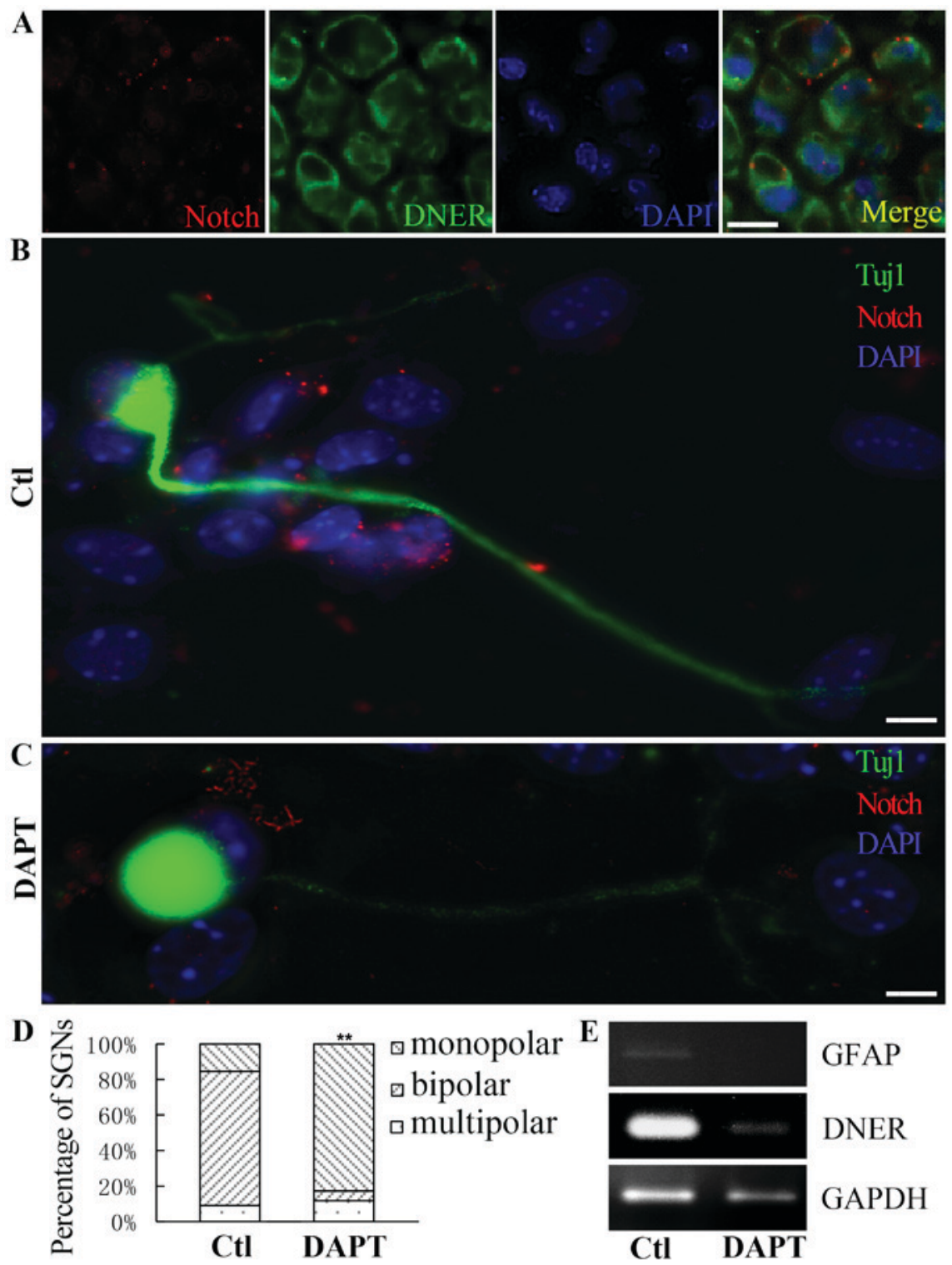

Figure 5. DAPT affected the polarity and the formation of protrusions in cultured neurons. (A) Notch expressed in SGNs from a P1 mouse. Scale bar, $40 \mu \mathrm{m}$. (B) In the absence of DAPT, the proportion of neural progenitors differentiating into active-Notch expressing bipolar neurons was greater. Scale bar, $5 \mu \mathrm{m}$. (C) Following DAPT treatment, the proportion of spiral ganglion progenitors differentiating into monopolar neurons was greater. Notch is presented in red, DNER is presented in green and nuclei are presented in blue. Scale bar, $5 \mu \mathrm{m}$. (D) The control group, in the absence of DAPT, was dominated by bipolar neurons, whereas, following DAPT treatment, the proportions of monopolar and multipolar neurons were higher. Mean \pm standard deviation; ${ }^{* *} \mathrm{P}<0.01$. (E) DAPT treatment resulted in reduced DNER and GFAP expression during neural progenitor differentiation. DAPT, N-[N-(3,5-Difluorophenacetyl)-L-alanyl]-S-phenylglycine t-butyl ester; SGN, spiral ganglion neuron; DNER, Delta/Notch-like epidermal growth factor-related receptor; GFAP, glial fibrillary acidic protein; Ctl, control.

compared with controls (Fig. 5D). Furthermore, DAPT treatment reduced the expression of DNER and GFAP during the development of spiral ganglion progenitors (Fig. 5E).

\section{Discussion}

Previous studies have demonstrated that DNER is specifically expressed during CNS development and maturation $(7,8)$ and important roles have been suggested for it in neural development $(9,10,12,18)$. In the developing cerebellum, DNER and Notch1 have been suggested to interact at Purkinje cell-Bergmann glia contacts (10). Notch signaling may be implicated in the control of the generation and differentiation of neuronal and glial cells through various pathways. It has been suggested that DNER-Notch signaling may be involved in the morphological differentiation of Bergmann glial cells (10). Treatment with DNER or activation of Notch signaling in Bergmann glia in $\mathrm{DNER}^{-/-}$mice has been reported to attenuate impairments in Bergmann fiber formation (10). In vitro, DNER has been demonstrated to specifically bind to Notch-expressing fusiform glial cells and induce process elongation to promote radial fiber formation in Bergmann glia, via a $\gamma$-secretase- and Deltex-dependent but cerebellar soluble lectin-independent Notch signaling pathway (10). Notably, the Deltex-dependent pathway was activated by either $\mathrm{F} 3$ neuronal adhesion molecule or DNER and was reported to induce terminal differentiation of glia after fate determination, whereas the canonical pathway was demonstrated to maintain an undifferentiated 
state. After binding to ligands such as Delta or Jagged, Notch undergoes two proteolytic cleavages by tumor necrosis factor- $\alpha$-converting enzyme (TACE) and $\gamma$-secretase-like proteinases that release its intracellular domain (NICD). The cleaved NICD translocates to the nucleus and forms a complex with CBF1 (RBP-J)/Su(H)/LAG1 (CSL) family transcription factors, which in turn activates transcription of Notch effector genes, including Hes genes. Therefore, DNER has been suggested to activate various pathways and responses depending on cell type and state, via Notch-dependent and Notch-independent mechanisms (9).

The present results, in accordance with previous studies, demonstrated that in the peripheral nervous system DNER was expressed in the somatoplasm of embryonic and adult SGNs, including their afferent and efferent nerve endings to cochlear hair cells. Furthermore, in the vestibule and cochlea, DNER expression has been reported in developing and mature hair cells and vestibular neurons (13). These findings suggested that DNER, which is widely expressed in the peripheral neurosensory epithelium, may serve important roles in its development and differentiation, similar to its roles in the CNS $(9,10)$. However, the role of DNER in spiral ganglion development and differentiation has yet to be elucidated. The present study demonstrated that DNER was expressed in the mouse inner ear, and its expression was temporospatially specific and polarized. During cochlear development, DNER expression was demonstrated to gradually increase, and marked polarity was sustained in adult neurons; however, DNER expression appeared evanescent in aged mice. Neuronal precursors from spiral ganglia cultured in vitro also exhibited similar characteristics and polar DNER distribution during neuronal differentiation, whereas the polarity disappeared during further culture. Therefore, it may be hypothesized that DNER is related to SGN polarity, as well as protrusion formation, dendritic growth and neuronal survival.

When the expression of DNER was silenced in neural progenitors isolated from neonatal mouse cochlea in vitro, the number of differentiated neurons significantly decreased, and fewer bipolar neurons with shorter neurites were present compared with the control group. Furthermore, the expression of $\alpha$-synuclein, which is involved in synaptic vesicle transport and exocytosis, and the GluR2/3 subunits of the synaptic $\alpha$-amino-3-hydroxy-5-methyl-4-isoxazolepropionic acid glutamate receptor were markedly reduced following DNER knockdown. These findings suggested that DNER may be involved in synapse formation. Similar findings were observed following treatment with the $\gamma$-secretase inhibitor DAPT, as it inhibitedNotch1 activation, and resulted in reduced DNER expression, as well as reduced neuronal differentiation and neurite growth. In accordance with previous studies $(9,10,13)$, the present results suggested that DNER may be able to induce neuronal and glial differentiation, and regulate neurite extension and growth $(9,21-24)$.

In conclusion, the present study demonstrated that DNER expression in developing SGNs was characterized by temporospatial specificity. The knockdown of DNER expression reduced the number of differentiated SGNs and impaired neuritogenesis. DNER appeared to be exerting its actions via a Notch-dependent signaling pathway. Therefore, the present results suggested that the DNER/Notch signaling pathway may serve important roles in spiral ganglion development and neuritogenesis.

\section{Acknowledgements}

The study was supported by grants from China Postdoctoral Science Foundation (grant no. 2014M562329), the National Natural Science Foundation of China (grant nos. 81271076, 81200748 and 81500802).

\section{References}

1. Xu HX, Kim GH, Snissarenko EP, Cureoglu S and Paparella MM: Multi-channel cochlear implant histopathology: Are fewer spiral ganglion cells really related to better clinical performance? Acta Otolaryngol 132: 482-490, 2012.

2. Roehm PC and Hansen MR: Strategies to preserve or regenerate spiral ganglion neurons. Curr Opin Otolaryngol Head Neck Surg 13: 294-300, 2005.

3. Petrovic J, Formosa-Jordan P, Luna-Escalante JC, Abelló G, Ibañes M, Neves J and Giraldez F: Ligand-dependent Notch signaling strength orchestrates lateral induction and lateral inhibition in the developing inner ear. Development 141: 2313-2324, 2014.

4. Kiernan AE: Notch signaling during cell fate determination in the inner ear. Semin Cell Dev Biol 24: 470-479, 2013.

5. Brooker R, Hozumi K and Lewis J: Notch ligands with contrasting functions: Jagged1 and Deltal in the mouse inner ear. Development 133: 1277-1286, 2006.

6. Haddon C, Jiang YJ, Smithers L and Lewis J: Delta-Notch signalling and the patterning of sensory cell differentiation in the zebrafish ear: Evidence from the mind bomb mutant. Development 125: 4637-4644, 1998.

7. Eiraku M, Hirata Y, Takeshima H, Hirano T and Kengaku M: Delta/notch-like epidermal growth factor (EGF)-related receptor, a novel EGF-like repeat-containing protein targeted to dendrites of developing and adult central nervous system neurons. J Biol Chem 277: 25400-25407, 2002.

8. Nishizumi H, Komiyama T, Miyabayashi T, Sakano S and Sakano H: BET, a novel neuronal transmembrane protein with multiple EGF-like motifs. Neuroreport 13: 909-915, 2002.

9. Hsieh FY, Ma TL, Shih HY, Lin SJ, Huang CW, Wang HY and Cheng YC: Dner inhibits neural progenitor proliferation and induces neuronal and glial differentiation in zebrafish. Dev Biol 375: 1-12, 2013

10. Eiraku M, Tohgo A, Ono K, Kaneko M, Fujishima K, Hirano T and Kengaku M: DNER acts as a neuron-specific Notch ligand during Bergmann glial development. Nat Neurosci 8: $873-880,2005$.

11. Saito SY and Takeshima H: DNER as key molecule for cerebellar maturation. Cerebellum 5: 227-231, 2006.

12. Tohgo A, Eiraku M, Miyazaki T, Miura E, Kawaguchi SY, Nishi M, Watanabe M, Hirano T, Kengaku M and Takeshima H: Impaired cerebellar functions in mutant mice lacking DNER. Mol Cell Neurosci 31: 326-333, 2006.

13. Hartman BH,Nelson BR, Reh TAandBermingham-McDonogh O: Delta/notch-like EGF-related receptor (DNER) is expressed in hair cells and neurons in the developing and adult mouse inner ear. J Assoc Res Otolaryngol 11: 187-201, 2010.

14. Kowalik L and Hudspeth AJ: A search for factors specifying tonotopy implicates DNER in hair-cell development in the chick's cochlea. Dev Biol 354: 221-231, 2011.

15. Fukazawa N, Yokoyama S, Eiraku M, Kengaku M and Maeda N: Receptor type protein tyrosine phosphatase zeta-pleiotrophin signaling controls endocytic trafficking of DNER that regulates neuritogenesis. Mol Cell Biol 28: 4494-4506, 2008.

16. Richardson L, Venkataraman S, Stevenson P, Yang Y, Moss J, Graham L, Burton N, Hill B, Rao J, Baldock RA and Armit C: EMAGE mouse embryo spatial gene expression database: 2014 update. Nucleic Acids Res 42 (Database issue): D835-D844, 2014.

17. Oshima K, Grimm CM, Corrales CE, Senn P, Martinez Monedero R, Géléoc GS, Edge A, Holt JR and Heller S: Differential distribution of stem cells in the auditory and vestibular organs of the inner ear. J Assoc Res Otolaryngol 8: 18-31, 2007. 
18. Zhao LD, Guo WW, Lin C, Li LX, Sun JH, Wu N, Ren LL, Li XX, Liu HZ, Young WY, et al: Effects of DAPT and Atoh1 overexpression on hair cell production and hair bundle orientation in cultured organ of corti from neonatal rats. PLoS One 6: e23729, 2011.

19. Sage C, Huang M, Karimi K, Gutierrez G, Vollrath MA, Zhang DS, Garcia-Añoveros J, Hinds PW, Corwin JT, Corey DP and Chen ZY: Proliferation of functional hair cells in vivo in the absence of the retinoblastoma protein. Science 307: 1114-1118, 2005.

20. Madden TA, Barrow D, McClelland RA, Gee JM and Nicholson RI: Modulation of oestrogen action by receptor gene inhibition. Eur J Cancer 36 (Suppl 4): S34-S35, 2000

21. Artavanis-Tsakonas S, Rand MD and Lake RJ: Notch signaling: Cell fate control and signal integration in development. Science 284: 770-776, 1999.
22. Berezovska O, McLean P, Knowles R, Frosh M, Lu FM, Lux SE and Hyman BT: Notch1 inhibits neurite outgrowth in postmitotic primary neurons. Neuroscience 93: 433-439, 1999.

23. Redmond L, Oh SR, Hicks C, Weinmaster G and Ghosh A: Nuclear Notch1 signaling and the regulation of dendritic development. Nat Neurosci 3: 30-40, 2000.

24. Redmond L and Ghosh A: The role of Notch and Rho GTPase signaling in the control of dendritic development. Curr Opin Neurobiol 11: 111-117, 2001.

c) (i) $(-)$ This work is licensed under a Creative Commons Attribution-NonCommercial-NoDerivatives 4.0 International (CC BY-NC-ND 4.0) License. 